\title{
MICROCHIP RFID PASIVO IMPLANTADO EN DIENTES MOLARES SOMETIDOS A ALTAS TEMPERATURAS CON FINES DE IDENTIFICACIÓN FORENSE
}

\author{
${ }^{1}$ Freddy Moreno Gómez, ${ }^{2}$ Sandra Milena Moreno Correa, ${ }^{3}$ Herney Garzón Rayo \\ ${ }_{1,2}$ Magíster en Ciencias Biomédicas U. del Valle, Docente U. Javeriana de Cali, Colombia. \\ ${ }^{3}$ Especialista en Rehabilitación Oral U. Militar Nueva Granada, Docente U. del Valle, Colombia.
}

Autor responsable de correspondencia: Freddy Moreno Gómez

Correo electrónico: fmorenog@javerianacali.edu.co

RESUMEN

Objetivo: valorar el comportamiento In vitro de un microchip RFID pasivo implantado en molares humanos y sometidos a altas temperaturas para determinar su capacidad de resistencia y funcionamiento.

Materiales y métodos: estudio experimental In vitro que evaluó el comportamiento de un microchip RFID pasivo (VeriChip ${ }^{\mathrm{TM}}$ ) implantado en diez molares humanos a través de una restauración en resina (Filtek $\mathrm{P}^{\mathrm{TM}}{ }^{\mathrm{TM}}$ Silorane $3 \mathrm{M}-\mathrm{ESPE}^{\circledR}$ ) en cavidades oclusales clase I y vestibulares clase V y sometido a altas temperaturas $\left(100{ }^{\circ} \mathrm{C}, 200{ }^{\circ} \mathrm{C}, 300{ }^{\circ} \mathrm{C}, 400{ }^{\circ} \mathrm{C}\right.$ y $\left.500{ }^{\circ} \mathrm{C}\right)$, con el fin de determinar su capacidad de resistencia y funcionamiento. Los especímenes fueron observados a través de Fotografia Digital (FD), Estereomicroscopio (EM) y Microscopio Electrónico de Barrido (MEB).

Resultados: se observó que los microchips RFID pasivos tienen capacidad de resistir a las altas temperaturas al conservar su integridad macroestructural; sin embargo, se observaron fracturas cohesivas y adhesivas que alteran los mecanismos de adhesión entre los tejidos dentales, la resina y el microchip, y el correcto funcionamiento de este último a partir de los $300^{\circ} \mathrm{C}$.

Conclusiones: los microchips RFID pasivos implantados en molares humanos a través de una restauración en resina resisten la acción de las altas temperaturas sin alterar su macroestructura. No obstante, solo funcionaron hasta los $300{ }^{\circ} \mathrm{C}$; lo que, eventualmente, podrá contribuir con los procesos de identificación forense para el caso de cadáveres quemados, carbonizados e incinerados. [Moreno $\mathrm{F}$, Moreno SM, Garzón H. Microchip RFID pasivo implantado en dientes molares sometidos a altas temperaturas con fines de identificación forense. Ustasalud 2013; 12: 116 - 123].

Palabras clave: Ciencias forenses, Odontología forense, Materiales biocompatibles.

\section{IMPLANTED PASSIVE RFID MICROCHIPS IN MOLARS SUBJECTED TO HIGH TEMPERATURES WITH FORENSIC IDENTIFICATION PURPOSES}

\section{ABSTRACT}

Objective: to assess In vitro behavior of a passive RFID microchip implanted in human molars and subjected to high temperatures to determine their resistance and performance.

Methods: an experimental In vitro study was done to evaluate the behavior of a passive RFID microchip (VeriChip ${ }^{\mathrm{TM}}$ ) implanted in 10 human molars with a composite restoration (Filtek $\mathrm{P} 90^{\mathrm{TM}}$ Silorane $3 \mathrm{M}-\mathrm{ESPE}^{\circledR}$ ) in class I occlusal cavities and class V vestibular cavities and subjected to high temperatures $\left(100{ }^{\circ} \mathrm{C}, 200{ }^{\circ} \mathrm{C}, 300^{\circ} \mathrm{C}, 400^{\circ} \mathrm{C}\right.$ and $\left.500{ }^{\circ} \mathrm{C}\right)$, in order to determine their resistance and performance. The specimens were observed through Digital Photography (DP), Stereomicroscopy (SM) and Scanning Electron Microscopy (SEM). Results: it was observed that passive RFID microchips have capacity to withstand high temperatures maintaining their macrostructural integrity, but they were observed cohesive and adhesive fractures that disrupt the mechanisms of adhesion between the dental tissues, the composite material and the microchip, and impairs its correct operation beyond $300{ }^{\circ} \mathrm{C}$.

Conclusions: passive RFID microchips implanted in human molars with a resin restoration resist the action of high temperatures without altering their macrostructure, however they only operated up to $300{ }^{\circ} \mathrm{C}$, which eventually may contribute to the processes of forensic identification in case of burned, charred and incinerated bodies.

Key words: Forensic sciences, Forensic dentistry, Biocompatible materials. 


\section{INTRODUCCIÓN}

En Colombia cada vez son más las muertes cuyo procedimiento de identificación se dificulta debido al estado del cuerpo o de los restos humanos (avanzado estado de descomposición, esqueletización, quemaduras, carbonización, incineración, mutilación, entre otros), como también a la alteración de tejidos blandos, la eliminación de las huellas digitales, la mala calidad de secuencias repetidas en tándem de ADN susceptibles de ser interpretadas y analizadas, o la ausencia de otros elementos que conlleven a una identificación positiva o fehaciente de un individuo. Es por ello, que las instituciones encargadas de dicho proceso de identificación deben contar con métodos más eficaces que permitan el rápido reconocimiento de un individuo, para cumplir, no solo con la labor social respecto a los familiares, sino también con la agilización del caso desde el punto de vista judicial. En la actualidad el uso de microchips RFID pasivos implantados en humanos se está popularizando con diferentes objetivos médicos y económicos, cuya función principal es identificar y suministrar cierta información de un individuo. Dicha implantación es sub-dérmica (generalmente en el dorso de la mano y en el antebrazo) y ha sido aprobada por el Departamento de Salud y Servicios Humanos de la Administración de Drogas y Alimentos de los Estados Unidos (FDA de acuerdo con las siglas en inglés), y regulada por la normativa ISO. El modelo de implantación que se expone en este estudio es en los dientes, a través de una cavidad restaurada con una resina compuesta (Figura 1).

\section{Empleo de dispositivos para identificación odontológica}

En la literatura especializada se pudieron encontrar varios reportes de mecanismos para marcar los diferentes aparatos de rehabilitación oral con fines de identificación como los de Harvey, Turner y colaboradores, Ryan y colaboradores, Berry y colaboradores, Cross Wolfaard, Ling, Reeson y Moya y colaboradores. ${ }^{1-8}$ Así mismo la Asociación Dental Americana (ADA) desarrolló un microdisco acrílico (azul para hombre y rosado para mujeres) de unos 3 a 4 milímetros de diámetro, el cual sería cementado en la superficie vestibular del primer molar superior derecho. Este dispositivo tendría grabado un código alfanumérico único que identificaría a cada individuo. ${ }^{9,10}$ Rajan y Julian indicaron el empleo de microchips con estos fines, ${ }^{11}$ para lo cual Millet y Jeannin realizaron un estudio en el que incorporaron un microchip a prótesis totales acrílicas mediante el sistema de lectura o escaneo de RIFD pasivos. ${ }^{12}$ En la actualidad la empresa Dentalax ${ }^{\circledR}$ comercializa un microchip y su lector para etiquetar prótesis removibles parciales y totales que contengan acrílico. ${ }^{13}$ En cuanto a la implantación de microchips dentro de los dientes, Theviessen y colaboradores realizaron un estudio In vitro en el cual implantaron varios microchips RFID pasivos de uso veterinario (EasyTrac-ID ${ }^{\circledR}$ ) en molares humanos y concluyeron que

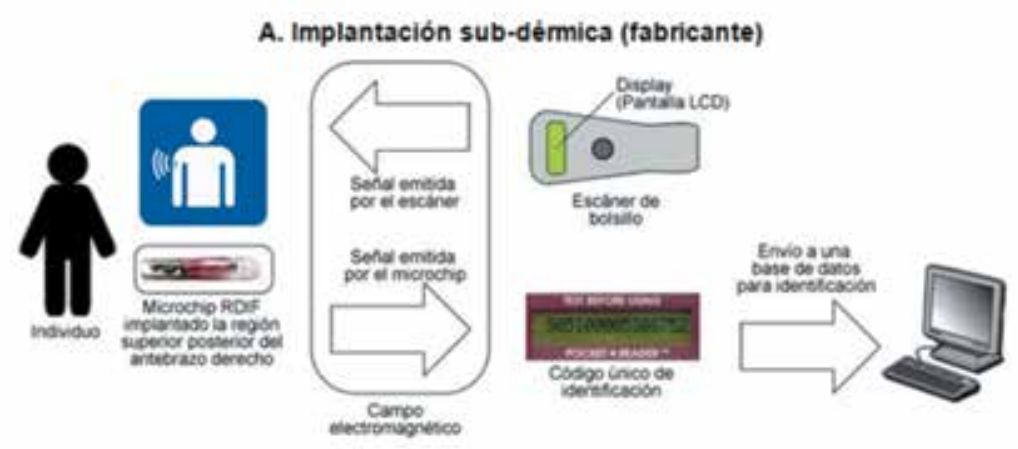

B. Implantación dental (propuesta de este estudio)
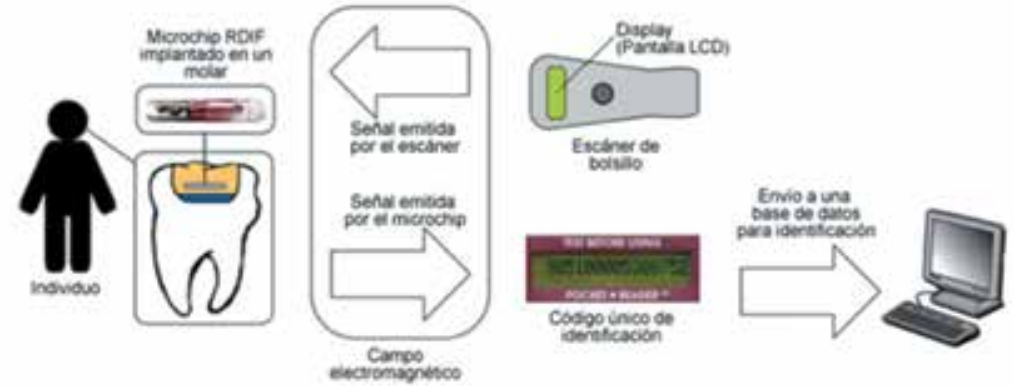

Figura 1. Implantación de los microchips (VeriChip ${ }^{\mathrm{TM}}$ ) 
este tipo de sistemas son de gran utilidad para la identificación de un individuo dentro del contexto forense. ${ }^{14,15}$

\section{Tejidos dentales y los materiales odontológicos a la ac-} ción de las altas temperaturas

Los tejidos dentales y los materiales de uso odontológico presentan diferentes comportamientos al ser sometidos a distintas temperaturas In vitro. ${ }^{16}$ Estos cambios varían de acuerdo con factores extrínsecos como el tiempo de exposición a la injuria térmica, la naturaleza de la causa del fuego, la participación de sustancias combustibles, la curva de elevación de la temperatura y las sustancias empleadas para extinguir el fuego y factores intrínsecos como el coeficiente de expansión térmico de los tejidos y los materiales, además del punto de fusión de estos últimos. Por otro lado, In situ, los dientes no se exponen de manera directa al fuego (sobre todo los dientes posteriores), por lo menos inicialmente, ya que se encuentran protegidos por los tejidos periorales, la musculatura facial y para el caso de las raíces la mucosa y el tejido óseo de los maxilares. Además, a medida que aumenta la temperatura del cuerpo, desde el sistema digestivo se expelen gases que llegan a la cavidad oral y proyectan la lengua en dirección postero-anterior provocando que los dientes queden inmersos en esta. ${ }^{17,18}$

En todo caso, los dientes son las estructuras más resistentes del cuerpo humano y resisten temperaturas de hasta $1000{ }^{\circ} \mathrm{C}$ sin pérdida importante de su microestructura, sobreviviendo casi intactos mucho tiempo después de que los tejidos blandos y esqueléticos se han destruido por incineración. ${ }^{19}$ Moya y colaboradores indicaron que por acción del calor, los tejidos dentales y los materiales de uso odontológico sufren alteraciones según la temperatura que se alcance, de la curva de elevación de la misma y del tiempo de exposición. ${ }^{8}$ Günther y Schmid -citados por Rötzscher y colaboradores- ${ }^{20}$ describieron los efectos del calor sobre los dientes y materiales empleados en rehabilitación oral y operatoria cuando se aplicaban altas temperaturas, siendo estas incrementadas en el transcurso de determinado tiempo. Nossintchoux -citado por Moya y colaboradores-,${ }^{8}$ describió alteraciones colorimétricas y estructurales de los dientes al ser sometidos a diferentes temperaturas. Así mismo, Moya y colaboradores y Andersen y colaboradores concluyeron que la alteración de materiales empleados en odontología por altas temperaturas depende del punto de fusión de los elementos que los componen. ${ }^{8,21}$ Merlati y colaboradores sometieron a altas temperaturas dientes intactos y con restauraciones y sugirieron que en diferentes grados de temperaturas se presentan cambios específicos en los tejidos dentales y en los materiales de uso odontológico. ${ }^{22,23}$ Esto fue corroborado por Savio y colaboradores a través de radiografias. ${ }^{24}$ Moreno y colaboradores describieron el comportamiento de los tejidos dentales y de algunos materiales de uso odontológico concluyendo que estos elementos presenta gran resistencia a la acción de las altas temperaturas. ${ }^{16}$ Esto fue corroborado en estudios posteriores por los mismos investigadores. ${ }^{25,26}$

\section{Microchip RFID}

Los microchips, etiquetas electrónicas, RFID tags o transponders de identificación electrónicos pasivos consisten en un artefacto de resonancia eléctrica conformado por un circuito de condensadores, una antena de recepción y transmisión, y un microchip electrónico, el cual, al entrar en contacto a través de la antena a una distancia de unos $10 \mathrm{~cm}$ aproximadamente con un campo electromagnético específico de baja potencia y Amplitud Modulada (AM) generado por el escáner, se alimenta con el voltaje inducido en la resonancia (de una frecuencia que va desde $125 \mathrm{kHz}$ hasta la banda ISM -Industrial Scientific and Medical- de 2,4 gHz, e incluso más), para que este envíe el código único de identificación al mismo escáner, una vez allí, el código se amplifica y se convierte en formato digital, de esta forma se descifra y se presenta el número único de identificación en la pantalla LCD del escáner. El tag consiste en el microchip en sí, el cual almacena un código de identificación de 16 dígitos, grabado en la superficie de forma inalterable con láser antes de su ensamble; la antena es una bobina de alambre de cobre alrededor de un núcleo de ferrita que se encarga de recibir y transmitir desde y hacia el lector las diferentes señales; y el condensador recibe del escáner el voltaje necesario para permitir que el microchip se active y emita el código de identificación.

\section{MATERIALES Y MÉTODOS}

Se realizó un estudio experimental In vitro para evaluar la resistencia a las altas temperaturas de diez microchips RFID pasivos (VeriChip ${ }^{\mathrm{TM}}$ ) implantados en diez molares humanos con el fin de determinar las posibilidades técnicas y clínicas de la implantación y guiar el protocolo de esta, respecto al diagnóstico del diente hospedero, al tamaño y la profundidad de la cavidad, a la selección del material de restauración dental y a la viabilidad de estos para resistir diferentes grados de temperatura.

\section{Recolección de la muestra}

Una vez obtenido el aval del Comité de Ética en Humanos de la Facultad de Salud, Universidad del Valle y de acuerdo con la Resolución 008430 y con la Declaración de Helsinki, ${ }^{27,28}$ y verificado el riesgo mí- 
nimo de este estudio, se recolectó la muestra de 10 dientes, obtenidos de los pacientes que asistieron a la Clínica de Cirugía Oral de la Escuela de Odontología de la Universidad del Valle y que requerían extracción de molares por motivos periodontales $\mathrm{u}$ ortodóncicos y que aceptaron firmar el consentimiento informado.

\section{Manejo y conservación de la muestra}

Una vez extraídos los dientes se procedió a lavarlos profusamente con agua de la llave para eliminar los restos de sangre y tejidos, y posteriormente se introdujeron en un recipiente oscuro de tapa hermética con la solución fijadora Cloramina $\mathrm{T}$ al 5\%. Los dientes permanecieron en Cloramina $\mathrm{T}$ por una semana y luego se colocaron en solución salina a temperatura ambiente de acuerdo con lo estipulado en la Norma ISO/DIS 11405:2003. ${ }^{29}$

\section{Distribución de la muestra}

Se conformaron cinco grupos de acuerdo con los grados de temperatura $\left(100^{\circ} \mathrm{C}, 200{ }^{\circ} \mathrm{C}, 300{ }^{\circ} \mathrm{C}, 400\right.$ ${ }^{\circ} \mathrm{C}$ y $500{ }^{\circ} \mathrm{C}$ ). En cada grupo se incluyeron dos dientes, uno con el microchip implantado en una cavidad oclusal clase I y otro con el microchip implantado en una cavidad vestibular clase V (Tabla 1).

\section{Preparaciones de los microchip RFID pasivos para ser implantados en la muestra}

Dado el tamaño del microchip (VeriChip ${ }^{\mathrm{TM}}$ ) empleado en este estudio, se hizo necesario reducir sus dimensiones con el fin de configurar una cavidad dental en la que quedara suficiente remanente dental resistente; que el microchip no entrara en contacto con las paredes de la cavidad (tejido dental) de tal forma que el material de restauración cubriera todo el microchip y pudiera cumplir con sus funciones físicas, biológicas y estéticas. Para ello, y de acuerdo con las indicaciones de Thevissen y colaboradores, ${ }^{14,15}$ se retiró el polímero poroso de polipropileno que recubre un extremo del microchip con un bisturí No. 15 y con fresas de diamante se eliminó la cápsula de vidrio (VeriChip Pocket Reader ${ }^{\mathrm{TM}}$ ), escaneando constantemente el microchip para comprobar su funcionamiento. Estos procedimientos disminuyeron las dimensiones del microchip de 13 a $9 \mathrm{~mm}$ de longitud y de 2,5 a $1,5 \mathrm{~mm}$ de diámetro.

\section{Preparación de las cavidades dentales}

A cinco dientes se les realizó una cavidad clase I y a cinco dientes una cavidad clase $\mathrm{V}$ con una pieza de alta velocidad con cuatro salidas de agua y alto torque (Kavo $7000 c^{\circledR}$ ) con refrigeración constante y fresas cilíndricas de diamante de grano medio para operatoria (Intensive Swiss ${ }^{\circledR}$ ). Las cavidades fueron realizadas a partir de la línea media oclusal y vestibular respectivamente, controladas por medio de topes de silicona dispuestos en la fresa y corroboradas con un calibrador electrónico.

\section{Obturación de las cavidades}

Se realizó una profilaxis con cepillo de profilaxis y una solución de bicarbonato de sodio, se secó la superficie con un papel absorbente y se procedió a emplear el sistema adhesivo $\mathrm{P}^{\circledR}{ }^{\circledR} 3 \mathrm{M}-\mathrm{ESPE}^{\circledR}$ (se aplicó el primero por 15 segundos, se aireó y se fotocuró por 15 segundos; luego se aplicó el adhesivo por 10 segundos, se aireó y se fotocuró resina por 10 segundos). Posteriormente, se colocó una primera capa de resina (Filtek $\mathrm{P}^{\mathrm{TM}}{ }^{\mathrm{TM}}$ Silorane $3 \mathrm{M}$-ESPE ${ }^{\circledR}$ ) en el fondo de la cavidad Clase I y Clase V, se puso el microchip y se fotocuró por 20 segundos. Para finalizar, se terminó la obturación de la cavidad me-

Tabla 1. Distribución de la muestra

\begin{tabular}{|c|c|c|c|}
\hline Temperatura & Diente & Serial del microchip & Tipo de cavidad \\
\hline \multirow{2}{*}{$100{ }^{\circ} \mathrm{C}$} & 1 & 1022000000050620 & Clase I \\
\hline & 2 & 1022000000033977 & Clase V \\
\hline \multirow{2}{*}{$200{ }^{\circ} \mathrm{C}$} & 3 & 1022000000026535 & Clase I \\
\hline & 4 & 1022000000050726 & Clase V \\
\hline \multirow{2}{*}{$300{ }^{\circ} \mathrm{C}$} & 5 & 1022000000040310 & Clase I \\
\hline & 6 & 1022000000027100 & Clase V \\
\hline \multirow{2}{*}{$400{ }^{\circ} \mathrm{C}$} & 7 & 1022000000029446 & Clase I \\
\hline & 8 & 1022000000031291 & Clase V \\
\hline \multirow{2}{*}{$500{ }^{\circ} \mathrm{C}$} & 9 & 1022000000034943 & Clase I \\
\hline & 10 & 1022000000036839 & Clase V \\
\hline
\end{tabular}


diante dos incrementos fotocurados cada uno por 20 segundos, se pulió y brilló la restauración con discos (Soflex ${ }^{\circledR} 3 \mathrm{M}-\mathrm{ESPE}^{\circledR}$ ) y a las restauraciones de las cavidades Clase I se les aplicó un agente sellador (Concise White Sealant 3M-ESPE ${ }^{\circledR}$ ) para sellar la unión de las cúspides. Para el proceso de la restauración se siguieron las indicaciones del fabricante ${ }^{30}$ y se escaneó (VeriChip Pocket Reader ${ }^{\mathrm{TM}}$ ) constantemente el microchip para comprobar su correcto funcionamiento. Los dientes fueron conservados en solución salina y rotulados con el serial del microchip hasta la aplicación de las pruebas de compresión.

\section{Aplicación de las altas temperaturas}

Una vez hechas las obturaciones, los dientes se llevaron a bandejas individuales confeccionadas con material de revestimiento refractario (Cera-Fina ${ }^{\circledR}$ Whipmix ${ }^{\circledR}$ ) para facilitar su manipulación de acuerdo con el prototipo patentado por la Unidad de Materiales Dentales del Departamento de Odontoestomatología de la Universidad de Pavia (Italia); y se sometieron al calor directo dentro de un horno tipo mufla (Thomas ${ }^{\circledR}$ Benchtop Industrial Furnace ${ }^{\circledR}$ ) previamente calibrado a cinco temperaturas (dos dientes a $100{ }^{\circ} \mathrm{C}$, luego dos dientes a $200{ }^{\circ} \mathrm{C}$, luego dos dientes a $300^{\circ} \mathrm{C}$, luego dos dientes a $400^{\circ} \mathrm{C}$ y luego dos dientes a $500{ }^{\circ} \mathrm{C}$ ) con una tasa de ascenso de $10^{\circ} \mathrm{C}$ por minuto desde una temperatura inicial de $34{ }^{\circ} \mathrm{C}$ (temperatura ambiente) hasta alcanzar cada una de las temperaturas propuestas. Terminada la aplicación de la temperatura específica, se retiraron los dientes del horno. Una vez fríos, se rociaron superficialmente con laca para cabello con el fin de conferirles cierto grado de resistencia y facilitar su manipulación. ${ }^{17}$

\section{Observación}

Una vez realizada la aplicación de las temperaturas se hizo el escaneo (VeriChip Pocket Reader ${ }^{\mathrm{TM}}$ ) de los microchips para comprobar su correcto funcionamiento y se procedió a realizar la toma de Fotografías Digitales (FD) a través de una Cámara Nikon ${ }^{\circledR}$ COOLPIX P6000 ${ }^{\circledR}$ del Departamento de Morfología de la Escuela de Ciencias Básicas de la Universidad del Valle. Posteriormente los especímenes fueron embebidos en acrílico trasparente (New Estethic ${ }^{\circledR}$ ) y se cortaron en un micrótomo de corte duro (Isomet Buheler ${ }^{\circledR}$ ) en sentido sagital (en sentido vestibularlingual) para exponer el sistema cavidad dental, resina y microchip y poder observar los cambios en los tejidos y materiales dentales a través de un estereomicroscopio (EM) trinocular Meiji ${ }^{\circledR}$ Techno RZ ${ }^{\circledR}$ del Departamento de Morfología de la Escuela de Ciencias Básicas de la Universidad del Valle; y de un microscopio electrónico de barrido (MEB) SEM $\mathrm{JEOL}^{\circledR}$ JSM 6490 LV $^{\circledR}$ de la Escuela de Ingeniería de
Materiales de la Facultad de Ingeniería de la Universidad del Valle.

La observación se realizó de acuerdo con el protocolo de descripción establecido por el Grupo de Investigación Cirugía Oral y Maxilofacial de la Universidad del Valle, al tener en cuenta la desadaptación de los materiales de obturación y del microchip (fallas cohesivas y adhesivas), las fisuras, grietas, aspecto cuarteado y fracturas, los cambios en el color y la textura, los niveles de carbonización e incineración. Todos ellos relacionados con el grado de temperatura al cual fueron sometidos los especímenes.

\section{RESULTADOS}

Los microchips sometidos a $100{ }^{\circ} \mathrm{C}, 200{ }^{\circ} \mathrm{C}$ y $300{ }^{\circ} \mathrm{C}$ (cavidad clase I) de temperatura funcionaron perfectamente, mientras que los microchips sometidos a $300{ }^{\circ} \mathrm{C}$ (cavidad clase V), $400{ }^{\circ} \mathrm{C}$ y $500{ }^{\circ} \mathrm{C}$ dejaron de funcionar luego de la exposición de la temperatura. Durante la manipulación de la muestra al aplicarse las altas temperaturas, un espécimen se perdió (Tabla 2).

\section{DISCUSIÓN}

En cuanto a la resistencia a la temperatura, la empresa que manufactura los microchips VeriChip ${ }^{\mathrm{TM}}$ Corporation) indica que estos soportan entre $40^{\circ} \mathrm{C}$ y $90^{\circ} \mathrm{C}$ en un tiempo constante de 1000 horas de almacenamiento, y entre $0^{\circ} \mathrm{C}$ y $50^{\circ} \mathrm{C}$ durante su funcionamiento. Pese a dicha información, Thevissen y colaboradores encontraron que los microchips funcionaban correctamente hasta los $300{ }^{\circ} \mathrm{C} ;{ }^{15} \mathrm{sin}$ embargo, manifiestan que durante la exposición de cada rango de temperatura, la lectura del microchip se interrumpía, lo cual fue relacionado con las corrientes de calor que se producen dentro del dispositivo. Luego de enfriarse, la lectura de los microchips resultó de nuevo posible. Dicho rango de temperatura fue corroborado en este estudio, en el que se evidenció que los microchips a $100^{\circ} \mathrm{C}$, $200{ }^{\circ} \mathrm{C}$ y $300^{\circ} \mathrm{C}$ (cavidad clase I) funcionaron perfectamente, mientras que los microchips a $300^{\circ} \mathrm{C}$ (cavidad clase V), $400{ }^{\circ} \mathrm{C}$ y $500{ }^{\circ} \mathrm{C}$ dejaron de funcionar luego de la exposición de la temperatura, debido a pérdida de propiedades estructurales de los tejidos dentales, de los materiales y del microchip mismo.

A los $100^{\circ} \mathrm{C}$, a través de las FD no se aprecian cambios significativos en los tejidos dentales y en la resina. En el EM se pueden observar microfracturas (fallas cohesivas) en el material de obturación, asociados con la expansión dimensional por las altas temperaturas. A través del MEB se pueden evidencia alteraciones microestructurales como la fractura de la cápsula de vidrio del microchip y en consecuencia fallas cohesivas entre el microchip y la resina. 
Tabla 2. Resultados de las pruebas a altas temperaturas

\begin{tabular}{|c|c|c|c|c|}
\hline Temperatura & Diente & Serial del microchip & Tipo de cavidad & Funcionamiento del microchip \\
\hline \multirow{2}{*}{$100{ }^{\circ} \mathrm{C}$} & 1 & 1022000000050620 & Clase I & - \\
\hline & 2 & 1022000000033977 & Clase V & $\nabla$ \\
\hline \multirow{2}{*}{$200{ }^{\circ} \mathrm{C}$} & 3 & 1022000000026535 & Clase I & $\nabla$ \\
\hline & 4 & 1022000000050726 & Clase V & $\nabla$ \\
\hline \multirow{2}{*}{$300{ }^{\circ} \mathrm{C}$} & 5 & 1022000000040310 & Clase I & $\nabla$ \\
\hline & 6 & 1022000000027100 & Clase V & $凶$ \\
\hline \multirow{2}{*}{$400{ }^{\circ} \mathrm{C}$} & 7 & 1022000000029446 & Clase I & 凶 \\
\hline & 8 & 1022000000031291 & Clase V & 凶 \\
\hline \multirow{2}{*}{$500{ }^{\circ} \mathrm{C}$} & 9 & 1022000000034943 & Clase I & 凶 \\
\hline & 10 & 1022000000036839 & Clase V & 凶 \\
\hline
\end{tabular}

- Microchip perdido, $\nabla$ Microchip funcional, $₫$ Microchip no funcional.

De igual forma se puede observar la integridad de las interfases adhesivas entre los tejidos dentales, el sistema adhesivo y la resina (Figura 2).

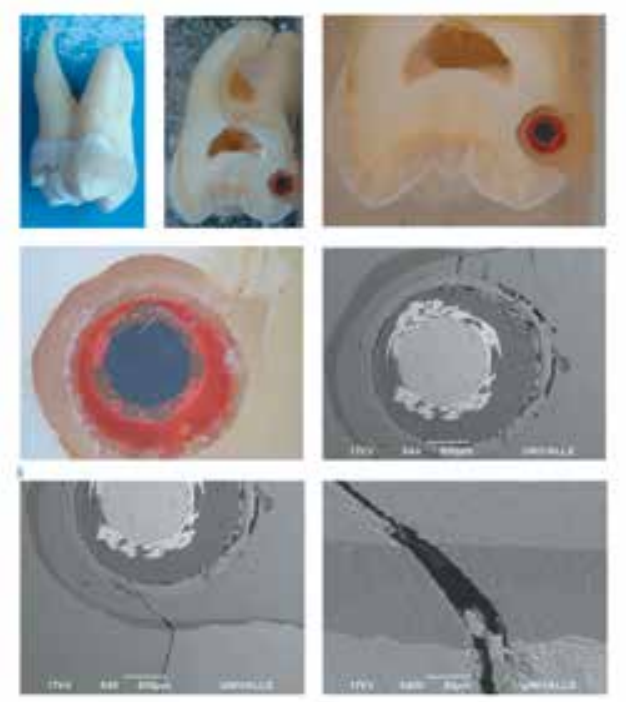

Figura 2. Diente con obturación clase $\mathrm{V}$ sometido a $100{ }^{\circ} \mathrm{C}$.

A los $200{ }^{\circ} \mathrm{C}$, las FD permiten observar que las coronas de los dientes pierden brillo asociado a la deshidratación del esmalte y la resina se torna opaca con vetas blancas debido al inicio de la combustión de la matriz acrílica. Ambas características fueron descritas en los estudios previos de Merlati y colaboradores, Moreno y colaboradores, y Vázquez y colaboradores. ${ }^{16,22,25,26}$ En los cortes sagitales observados en el EM se pueden apreciar los cambios de color en el esmalte más superficial y en toda la extensión de la unión del esmalte con la dentina (situación que le confiere ese color a la corona al ser el esmalte traslúcido), la cual se torna parda. Además resulta evidente la fractura de la cápsula de vidrio del microchip y las fallas adhesivas entre esta estructura y la resina. En el MEB todas estas alteraciones son magnificadas del mismo modo que se pueden apreciar fracturas en la resina asociadas con el estrés de contracción que se continúan en la dentina (Figura 3).
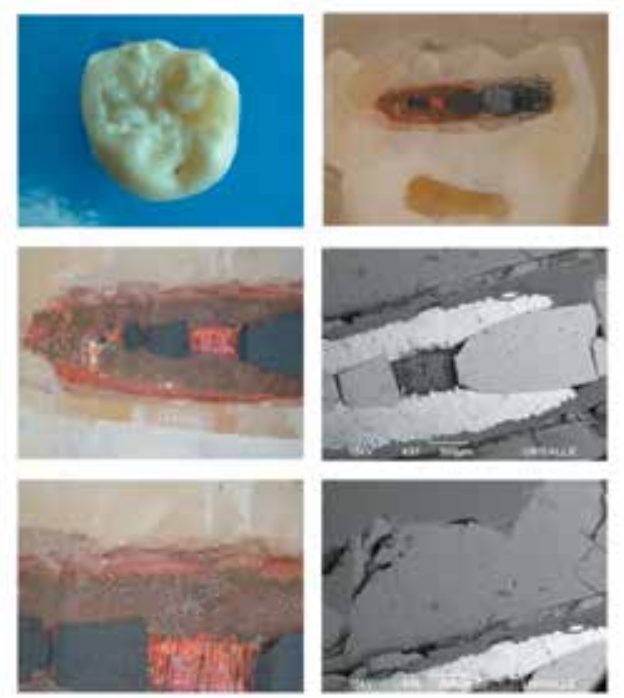

Figura 3. Diente con obturación clase I sometido a $200{ }^{\circ} \mathrm{C}$.

A los $300{ }^{\circ} \mathrm{C}$ en la corona se observan los rebordes marginales y cuspídeos totalmente blancos debido a la incineración de la matriz orgánica del esmalte. Del mismo modo se observan fracturas (fallas cohesivas) en el esmalte y en la interfase de esmalte y la resina (fallas adhesivas). La resina cambia de color a un tono pardo debido a la combustión de la matriz acrílica y presenta fallas cohesivas (fracturas en la resina misma) y fallas adhesivas (separación en la interfase esmalte y resina en el borde cavo de la cavidad asociado a la retracción marginal del material de obturación). En el EM y en el MEB, se pueden observar toda una serie de fallas adhesivas y cohesivas entre y de los tejidos dentales, la resina y el microchip, las cuales, causaron el daño estructural del microchip implantado en la cavidad clase V (Figura 4). 

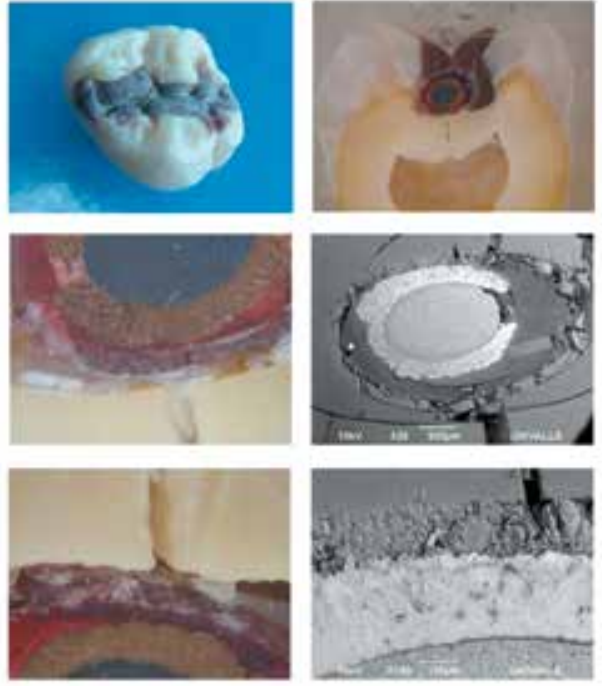

Figura 4. Diente con obturación clase I sometido a $300{ }^{\circ} \mathrm{C}$.

A los $400{ }^{\circ} \mathrm{C}$ en las FD se observa que el esmalte se encuentra opaco, evidencia microfracturas (aspecto cuarteado) y trasluce una dentina parda oscura producto de la carbonización de la matriz orgánica. La resina cambia de color a un pardo mucho más oscuro y presenta fallas cohesivas y adhesivas más evidentes que en el anterior grado de temperatura dentina, asociadas a la combustión de la matriz acrílica y a la alteración de la estabilidad dimensional. Estos cambios son coherentes con los descritos por Merlati y colaboradores, Moreno y colaboradores, y Vázquez y colaboradores en sus estudios. ${ }^{16,22,25,26}$ La observación en el EM permite evidenciar los cambios producto de la carbonización de la dentina, y en MEB se pueden apreciar todas las alteraciones en los tejidos dentales, en la resina y en el microchip, las cuales se describen como fallas adhesivas y cohesivas producto de la contracción dimensional asociada a las altas temperaturas (Figura 5).
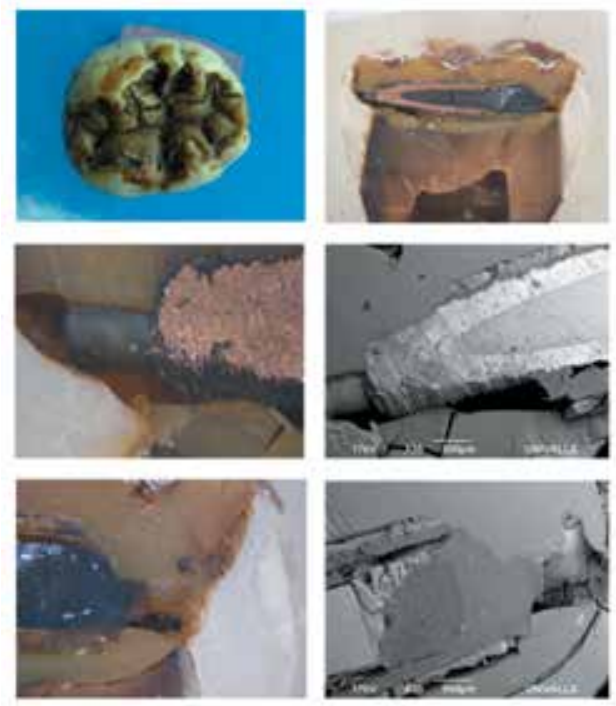

Figura 5. Diente con obturación clase I sometido a $400{ }^{\circ} \mathrm{C}$.
A los $500{ }^{\circ} \mathrm{C}$ la FD permite observar que la corona adopta un color café intenso relacionado con la carbonización de la dentina subyacente y el esmalte, de aspecto cuarteado y con grietas longitudinales, se separa de la dentina en la región cervical. La resina, de color negro debido a una carbonización completa, se encuentra desadaptada de la cavidad (fallas adhesivas) con múltiples fisuras y grietas superficiales (fallas cohesivas). En el EM la dentina, fisurada y agrieta$\mathrm{da}$, se ve carbonizada. Producto de estas alteraciones se observa pérdida de continuidad en las interfases entre los tejidos dentales y la resina, y el microchip y la resina. Lo cual se puede apreciar en detalle en el MEB, en donde además se puede apreciar la desintegración casi total del agente adhesivo, lo cual deja una brecha entre los tejidos dentales y la resina (Figura 6).
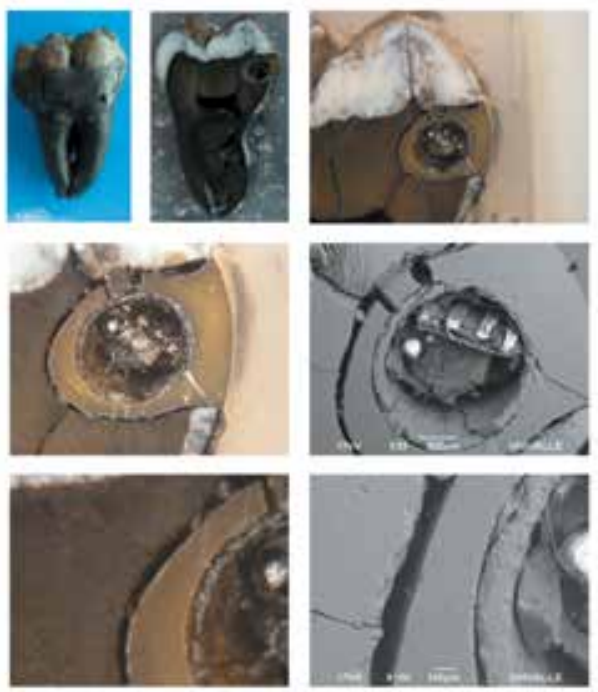

Figura 6. Diente con obturación clase V sometido a $500{ }^{\circ} \mathrm{C}$.

\section{Conclusiones}

El sistema cavidad dental, material de restauración y microchip, tiene gran resistencia estructural a las altas temperaturas. Funcionalmente, los microchips expuestos a $100{ }^{\circ} \mathrm{C}, 200{ }^{\circ} \mathrm{C}$ y $300{ }^{\circ} \mathrm{C}$ (cavidad clase I) emitieron su señal perfectamente, mientras que los microchips a $300^{\circ} \mathrm{C}$ (cavidad clase V), $400{ }^{\circ} \mathrm{C}$ y $500{ }^{\circ} \mathrm{C}$ dejaron de funcionar luego de la exposición de la temperatura. Por lo tanto, un microchip implantado en una cavidad clase I o clase V y obturado con un material de restauración de resina, se constituye en un adecuado sistema de identificación de individuos que resulten quemados, carbonizados 0 incinerados; teniendo en cuenta que si bien el microchip, no transmite señal a partir de los $300{ }^{\circ} \mathrm{C}$, la implantación per se, puede ser considerada como un marcador, inicialmente indiciario, a la hora de realizar el cotejo antemortem-postmortem.

\section{Agradecimientos}

Esta investigación fue financiada por la Vicerrectoría de Investigaciones de la Universidad del Valle a través de la 
Convocatoria Interna para la Conformación del Banco de Proyectos de Investigación - 2008.

\section{BIBLIOGRAFÍA}

1. Harvey W. Identity by teeth and the marking of dentures. Br Dent J. 1966; 121: 334 - 340.

2. Turner $\mathrm{CH}$, Fletcher AM, Ritchie GM. Denture marking and human identification. Br Dent J. 1976; 141: 114 - 117.

3. Ryan LD, Keller JB, Rogers DE, Schaeffer L. Clear acrylic resin T-bar used in denture identification. J Prosthet Dent. 1993; 70: 189 - 190.

4. Berry FA, Logan GI, Plata R, Riegel R. A post fabrication technique for identification of prosthetic devices. J Prosthet Dent. 1995; 73: 341 - 343.

5. Cross P, Wolfaardt JF. Denture identification system. J Prosthet Dent. 1995; 74: 551 - 552.

6. Ling BC. Computer-printer denture microlabeling system. J Prosthet Dent. 1998; 79: 363 - 364.

7. Reeson MG. A simple and inexpensive inclusion technique for denture identification. J Prosthet Dent. 2001; 86: $441-442$.

8. Moya V, Roldan B, Sánchez JA. Materiales dentales en la identificación. En Odontología Legal y Forense. Barcelona: Editorial Masson S. A.; 1994. p. 269 - 276.

9. Gladfelter IA, Smith BE. An evaluation of microdisk for dental identification. J Prosthetic Dentistry. 1989; 6: 352 - 355.

10. Hansen RW. Intraoral micro-identification discs. J Forensic Odontostomatol. 1991; 9: 77 - 91.

11. Rajan M, Julian R. A new method of marking dentures using microchips. J Forensic Odontostomatol. 2002; 20:1 - 5.

12. Millet $\mathrm{C}$, Jeannin $\mathrm{C}$. Incorporation of microchips to facilitate denture identification by radio frequency tagging. J Prosthet Dent. 2004; 92: 588 - 590.

13. Dentalax Coorporation (sede Web). 2006 [Fecha de consulta: 31 de Julio de 2013]. Disponible en http://dawhois. com/www/dentalax.com.html

14. Thevissen PW, Poelman G, De Cooman M, Puers R, Willems G. Implantation of an RFID-tag into human molars to reduce hard forensic identification labor. Part I: Working principle. Forensic Sci Int. 2006; 159: 33 - 39.

15. Thevissen PW, Poelman G, De Cooman M, Puers R, Willems G. Implantation of an RFID-tag into human molars to reduce hard forensic identification labor. Part 2: Physical properties. Forensic Sci Int. 2006; 159: 40 - 46.

16. Moreno S, León ME, Marín L, Moreno F. Comportamiento de los tejidos dentales y de algunos materiales de obturación dental sometidos a altas temperaturas con fines forenses. Colomb Med. 2008; 39: 28 - 46.

17. Delattre VF. Burned beyond recognition: Systematic approach to the dental identification of charred human remains. J Forensic Sci 2000; 45: 589 - 596.

18. Ferreira JL, Espina AL, Barrios FA, Mavaréz MG. Conservación de las estructuras orales y faciales del cadáver quemado. Ciencia Odontológica. 2005; 2: 58 - 65.

19. Norrlander AL. Burned and incinerated remains. En: Bowers CM, Bell GL, Editors. Manual of Forensic Odontology. 3rd Edition. Colorado Springs: American Society of Forensic Odontology; 1997. p. 16 - 18.

20. Rötzscher K, Grundmann C, Benthaus S. The effects of high temperatures on human teeth and dentures. Int Poster J Dent Oral Med. 2004; 6: Poster 213.
21. Andersen L, Juhl M, Solheim T, Borrman H. Odontological identification of fire victims - potentialities and limitations. Int J Leg Med. 1995; 107: 229 - 232.

22. Merlati G, Savio C, Danesino P, Fassina G, Menghini P. Further Study of restored and unrestored teeth subjected to high temperatures. J Forensic Odontostomatol. 2004; 22: 17-24.

23. Merlati G, Danesino P, Savio C, Fassina G, Osculati A, Menghini P. Observations of dental prostheses and restorations subjected to high temperatures: experimental studies to aid identification processes. J Forensic Odontostomatol. 2002; 20: 17 - 24.

24. Savio C, Merlati G, Danesino D, Fascina G, Menghini P. Radiographic evaluation of teeth subjected to high temperatures: Experimental study to aid identification processes. Forensic Sci Int. 2006; 158: 108 - 116.

25. Moreno S, Marín L, Merlati G, Savio C, Moreno F. Effects of high temperatures on different dental restorative systems: experimental study to aid identification processes. J Forensic Dent Sci. 2009; 1: 17 - 23.

26. Vázquez L, Rodríguez P, Moreno F. Análisis macroscópico In vitro de los tejidos dentales y de algunos materiales dentales de uso en endodoncia, sometidos a altas temperaturas con fines forenses. Revista Odontológica Mexicana 2012; 16: 171 - 181.

27. Ministerio de Salud. Resolución № 008430 del octubre 4 de 1993. Por la cual se establecen las normas científicas, técnicas y administrativas para la investigación en salud. [Fecha de consulta: 31 de Julio 2013]. URL disponible en: http://www.urosario.edu.co/urosario_files/a2/a24fb07af561-4fcc-b611-affff4374bb7.pdf

28. Asociación Médica Mundial. Principios éticos para las investigaciones médicas en seres humanos. Declaración de Helsinki. Finlandia, junio 1964 [Fecha de consulta: 31 de Julio de 2013]. URL disponible en: http://www.wma.net/ es/30publications/10policies/b3/

29. International Organization of Standardization. Dental materials: Testing of adhesion to tooth structure. Ginebra: ISO/TS 11405; 2003.

30. 3M ESPE. Filtek Silorane Technical Profile: Low Shrink Posterior Restorative. Country; 2007. [Fecha de consulta: 31 de Julio de 2013]. URL disponible en: http:// multimedia. $3 \mathrm{~m} . \mathrm{com} / \mathrm{mws} /$ mediawebserver?mwsId = SSS SSu7zK1fslxtUNY_9M8_Sev7qe17zHvTSevTSeSSSSSS--

\section{Correos electrónicos de los autores:}

Freddy Moreno Gómez: fmorenog@javerianacali.edu.co Sandra Milena Moreno Correa: smmoreno@javerianacali.edu.co Herney Garzón Rayo: herney.garzon@univalle.edu.co 